\title{
Cyclooxygenase-2 Expression in Gastric Antral Mucosa Before and After Eradication of Helicobacter pylori Infection
}

\author{
Conor J. McCarthy, M.D., M.R.C.P.I., Leslie J. Crofford, M.D., Joel Greenson, M.D., and \\ James M. Scheiman, M.D., F.A.C.P., F.A.C.G. \\ Department of Internal Medicine, Divisions of Rheumatology and Gastroenterology, and Department of \\ Pathology, University of Michigan Medical Center, Ann Arbor, Michigan
}

\begin{abstract}
OBJECTIVE: Helicobacter pylori (H. pylori) causes chronic gastritis. The inducible prostaglandin synthetase cyclooxygenase 2 (COX-2) plays an important role in inflammatory conditions. We hypothesized that $H$. pylori-associated chronic gastritis would express COX-2 protein. Our aim was to evaluate the effect of eradication of $H$. pylori infection on COX-2 expression in the antral mucosa of patients before and after antibiotic therapy.
\end{abstract}

METHODS: Tissues were obtained from patients with nonulcer dyspepia undergoing $H$. pylori eradication. Ten patients with proven $H$. pylori infection and subsequent successful eradication were studied. Three biopsies of antral mucosa were evaluated before and after $H$. pylori eradication. The amount of acute and chronic inflammation was quantitated. Immunohistochemical staining for COX-2 was expressed as a percentage of the total number of cells and correlated with the degree of chronic inflammation.

RESULTS: Specific immunostaining for COX-2 was observed in antral mucosa of patients infected with $H$. pylori. Patchy cytoplasmic staining was seen in surface epithelial cells and strong cytoplasmic staining for COX-2 was seen in parietal cells. Spotty cytoplasmic staining for COX-2 was also seen in lamina propria plasma cells, as well as there being macrophages present in the germinal centers of lymphoid aggregates. COX-2 expression could be detected both before and after eradication of $\mathrm{H}$. pylori. The mean percentage of cells staining for COX-2 was significantly higher in H. pylori-infected mucosa, compared with mucosa after successful $H$. pylori eradication $(33.4 \% \pm 5.4$ vs $18.9 \% \pm$ $3.3, p=0.038)$. COX-2 immunostaining correlated best with the chronic inflammation score $\left(\mathrm{r}^{2}=0.78, p<0.001\right)$. There was a strong correlation for those subjects who were H. pylori infected, as well as for those who had successful H. pylori eradication.

CONCLUSIONS: $H$. pylori associated acute and chronic antral inflammation was associated with immunohistochemical detection of COX-2 protein in epithelial cells, in addition to associated mononuclear cells and parietal cells. Expression was reduced, but not eliminated, in the epithelium after successful eradication of $H$. pylori. Despite the reduction in COX-2 expression after $H$. pylori eradication, expression of COX-2 in epithelial cells remained and strongly correlated with the extent of the chronic inflammatory cell infiltrate. The clinical implications of $H$. pylori-associated induction of COX-2 expression for patients on selective COX-2 inhibitors, in addition to the role of COX-2 in gastric carcinogenesis, deserve further study. (Am J Gastroenterol 1999; 94:1218-1223. (C) 1999 by Am. Coll. of Gastroenterology)

\section{INTRODUCTION}

Helicobacter pylori (H. pylori) infection causes a characteristic local inflammatory response in the gastric mucosa, leading to a chronic gastritis. Although the majority of individuals have a clinically silent infection, diverse outcomes of the infection have been identified. These include not only the development of peptic ulcer disease, but also an increased risk of neoplastic transformation in the stomach, including noncardia adenocarcinoma and mucosa-associated lymphoid tissue lymphoma (MALTomas). This organism clearly has had a significant impact on the management of many upper gastrointestinal disorders. The public health debate regarding the role of widespread eradication of $H$. pylori infection remains ongoing.

Prostaglandins play an important physiological role in the protection of the upper gastrointestinal (GI) tract mucosa against injurious agents. Until recently, prostaglandin production in the gastroduodenal mucosa was thought to be due to a single isoform of cyclooxygenase (1). Recognition of an inducible isoform, cyclooxygenase-2 (COX-2), which plays a critical role for prostaglandin (PG) synthesis in inflammatory states, raises a question regarding the contribution of the various COX isoforms in mucosal prostaglandin levels. Analogous to the observation that other chronically inflamed tissues express increased amounts of COX-2 protein (2), H. pylori-associated chronic gastritis would be expected to express COX-2 protein, at least in infiltrating inflammatory cells. Although COX-2 is not present at significant 
levels in normal gastric mucosa (3), a recent report has suggested that COX-2 expression was increased at the edges of experimental gastric ulcers created in laboratory animals, and that COX-2 may be the source of prostaglandins important for ulcer healing (4). The role of COX-2 in inflamed gastric tissue is uncertain; however, if COX-2 has an essential role in healing, this may have important clinical implications for the potential clinical impact of highly selective COX-2 inhibitors currently in development for the management of arthritis. Although these agents do not appear to induce GI toxicity, their effect on prostaglandins potentially important in ulcer healing may have detrimental actions in patients with established peptic ulcer disease.

The International Agency for Research on Cancer has classified $H$. pylori as a group I carcinogen, a definite cause of gastric cancer in humans (5). Although the organism does not invade the gastric epithelium, bacterial factors lead to an intense inflammatory cell infiltrate with associated cytokine production, which perpetuates inflammation. $H$. pylori also induces inflammatory cells to produce reactive oxygen metabolites, which may damage DNA and promote carcinogenesis. Bacterial products, in combination with cytokines, lead to increases in basal and stimulated gastrin levels. In some patients, H. pylori-induced gastritis extends proximally from the antrum, where it is typically seen, to the body of the stomach. There, inflammation of the glandular stomach leads to chronic atrophic gastritis, low acid production despite hypergastrinemia, and a decreased risk of ulcer disease, but an increased risk for gastric malignancy (both adenocarcinoma and lymphoma). Because the induction of COX-2 expression has been shown to have an important role in the progression of neoplastic transformation in the colon, it may also play a role in the development of $\mathrm{H}$. pylori-associated gastric neoplasia. Indeed, COX-2 expression has been demonstrated in human gastric carcinoma tissue by immunohistochemistry (6).

Because H. pylori-associated changes in COX-2 expression in the gastric mucosa have implications for both peptic ulcer disease and neoplasia, we sought to evaluate the COX-2 expression in the antral mucosa of patients before and after eradication of $H$. pylori infection. We also correlated this expression with the histological changes of inflammation.

\section{MATERIALS AND METHODS}

\section{Patients}

Tissues were obtained from patients with nonulcer dyspepia undergoing $H$. pylori eradication in a randomized controlled trial (7). Patients with nonulcer dyspepia (normal endoscopy) received bismuth subsalicylate $524 \mathrm{mg}$ q.i.d. for $4 \mathrm{wk}$ with metronidazole $250 \mathrm{mg}$ q.i.d. for the first $2 \mathrm{wk}$ of therapy (eradication rate $=68 \%$ ). The success of antibiotic therapy was assessed by histology 4 wk after completion of therapy. Ten patients with nonulcer dyspepsia with proven $H$. pylori infection and subsequent successful eradication comprised the study group. Three biopsies of antral mucosa were evaluated immunohistochemically for the presence of COX-2 protein expression before and after successful eradication of $H$. pylori infection.

\section{Immunohistochemistry}

Immunohistochemical staining for COX-2 was performed using the Vectastain ABC-AP kit (Vector Laboratories, Burlingame, CA). The sections were deparaffinized, then preincubated with $0.5 \%$ bovine serum albumin (BSA) in PBS and diluted normal goat serum (1:66.7) for $20 \mathrm{~min}$. Sections were then incubated with antibody to COX-2 (Cayman Chemical, Ann Arbor, MI) diluted 1:100 in 0.5\% BSA in PBS or normal rabbit IgG overnight at $4^{\circ} \mathrm{C}$. This rabbit polyclonal IgG antihuman COX-2 antibody has no crossreactivity with sheep seminal vesical COX-1 (data on file, Cayman Chemical). The antibody also demonstrated no crossreactivity with human COX-1 in Western blots in our laboratory (data not shown). Sections were washed three times in PBS, then incubated for $1 \mathrm{~h}$ with goat antirabbit $\operatorname{IgG}$ at a dilution of 1:1000. Sections were further washed and incubated with an avidin and biotinylated alkaline phosphatase complex for $30 \mathrm{~min}$. Finally, the sections were washed and color was developed by immersion in alkaline phosphatase substrate solution (Vector Laboratories) for $1 \mathrm{~h}$. Sections were rinsed in water and counterstained with hematoxylin. Positive staining was indicated by a red color. Control stains with normal rabbit IgG were uniformly negative.

\section{Histological Scoring}

The presence and amount of acute and chronic inflammation were evaluated in 20 hematoxylin-and-eosin-stained formalin-fixed paraffin-embedded gastric biopsies from 10 patients. Chronic inflammation was defined as plasma cells in the lamina propria. The number of plasma cells in the lamina propria was graded semiquantitatively from 1-4, with 1 being a small number of plasma cells and 4 being a marked number. Acute inflammation was defined as neutrophils infiltrating gastric epithelial cells. Acute inflammation was graded semiquantitatively from $1-3$. Grade 1 was one focus of activity per biopsy, 2 was $2-3$ foci per biopsy, and 3 was $>3$ foci per biopsy, representing intense acute inflammation.

Immunohistochemical staining for COX-2 was assessed by counting the number of positive and negative staining cells on the surface of each biopsy. The pathologist was blinded to the $H$. pylori status of the patient and, as the slides were not counterstained, the degree of inflammation or presence of bacteria was difficult to discern. Cell counting was performed on the strongest staining region of each biopsy. Cell counts were restricted to the surface epithelium because the vast majority of positively staining cells were 

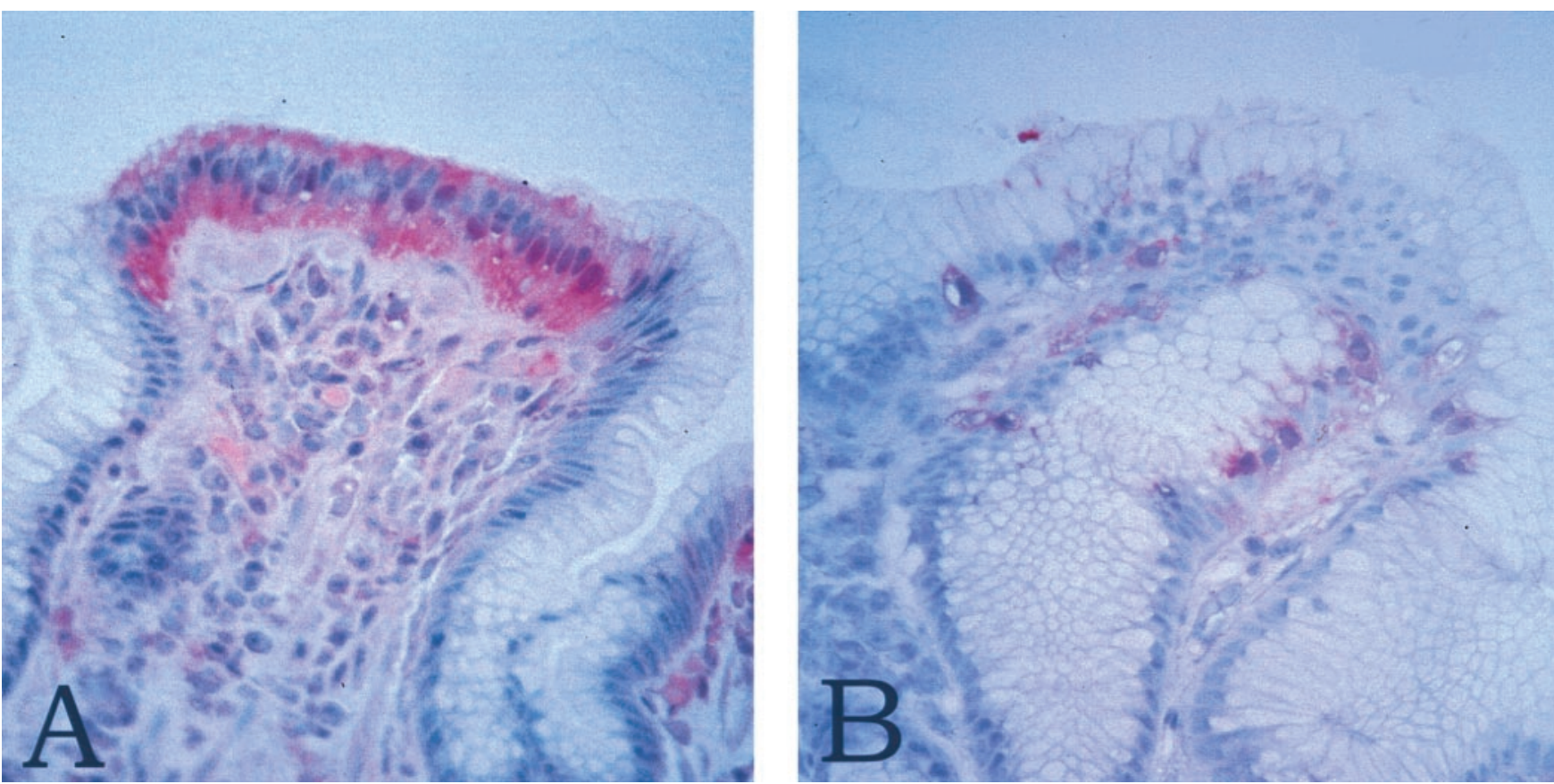

Figure 1. (A) Medium-power photomicrograph showing a diffuse area of positive staining for COX-2 in surface epithelial cells from a patient with untreated $H$. pylori gastritis (original magnification $\times 235$ ). (B) Medium-power photomicrograph showing a spotty distribution of COX-2-positive gastric surface cells from a patient with treated $H$. pylori gastritis (original magnification $\times 235$ ).

seen in this region. Inflammatory cells were not counted. Between 200 and 250 surface epithelial cells were counted in each biopsy. The number of positive staining cells was expressed as a percentage of the total number of cells.

\section{Statistical Analysis}

Comparison of COX-2 immunostaining in H. pylori-infected and -eradicated antral mucosa was analyzed using the Wilcoxon matched paired Signed-Rank test. Pearson's correlation coefficients were calculated to analyze the relationship between the percentage of COX-2 immunostaining and the acute and chronic inflammation scores.

\section{RESULTS}

Specific immunostaining for COX-2 was observed in the antral mucosa of patients infected with H. pylori (Fig. 1). Patchy cytoplasmic staining was seen in surface epithelial cells (Fig. 1). Strong cytoplasmic staining for COX-2 was seen in parietal cells in all cases where parietal cells were present. Spotty cytoplasmic positivity for COX-2 was also seen in lamina propria plasma cells, as well as in macrophages present in the germinal centers of lymphoid aggregates (Fig. 2). COX-2 positivity was not seen in the lymphocytes populating these lymphoid aggregates.

COX-2 expression could be detected both before and after eradication of $H$. pylori. The mean percentage of cells staining for COX-2 was significantly higher in $\mathrm{H}$. pyloriinfected mucosa compared with mucosa after successful $H$. pylori eradication $(33.4 \% \pm 5.4$ vs $18.9 \% \pm 3.3, p=0.038)$

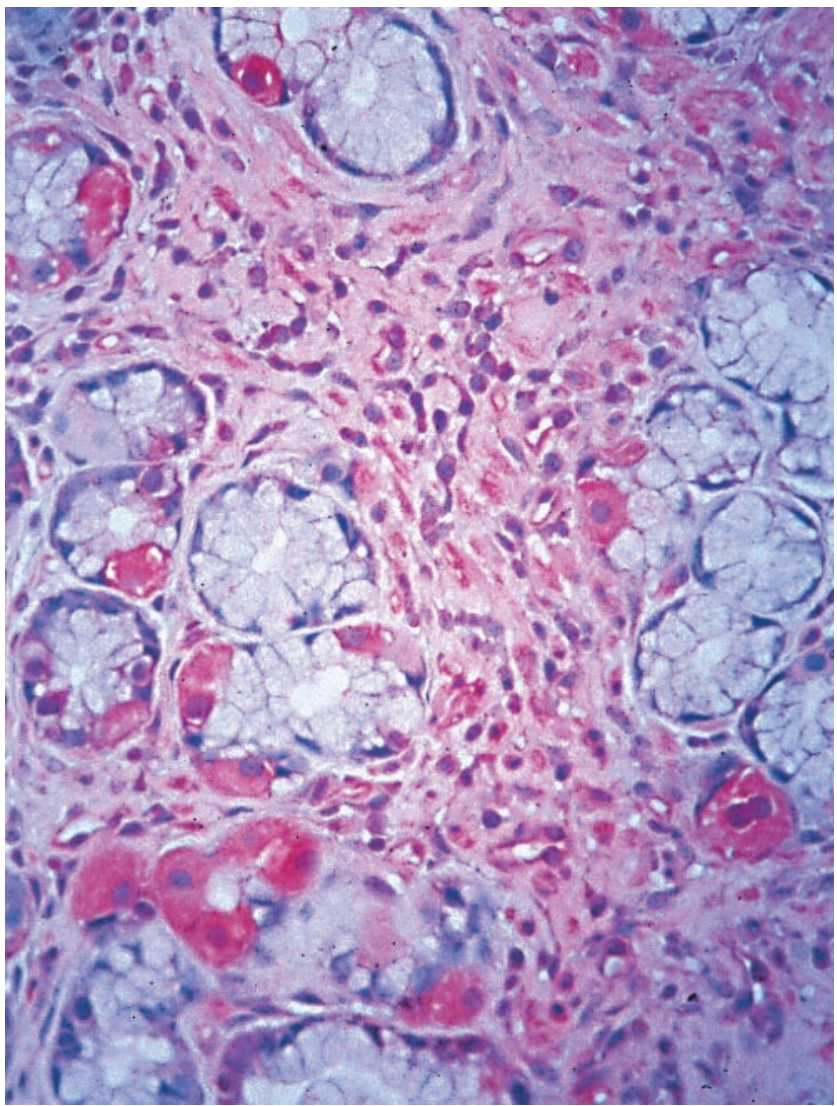

Figure 2. Medium-power photomicrograph showing COX-2 staining of parietal cells and plasma cells (original magnification $\times$ 235). 


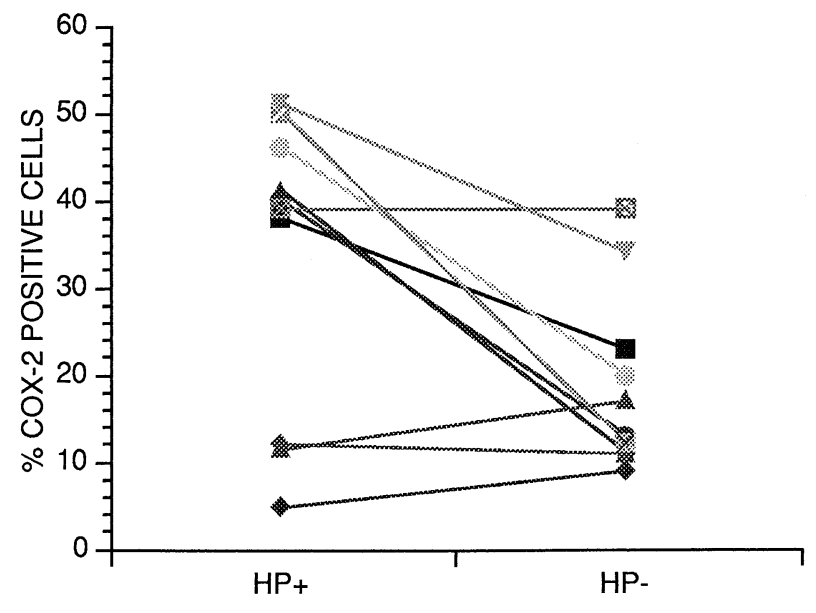

Figure 3. Reduction in COX-2 expression in gastric antral mucosa before and after eradication of $H$. pylori. The mean percentage of cells staining for COX-2 was significantly higher in $H$. pylori+ compared with $H$. pylori- patients $(33.4 \% \pm 5.4$ vs $18.9 \% \pm 3.3$, $p=0.038$ ).

(Fig. 2). Individual patients were examined before and after eradication of $\mathrm{H}$. pylori. There was a reduction of COX-2 expression in seven of 10 patients, no change in one, and a slight increase in two patients (Fig. 3). Acute inflammation resolved in all patients after eradication of $H$. pylori (Table $1)$, but was not well correlated with COX-2 expression $\left(\mathrm{r}^{2}=\right.$ $0.47, p=0.17)$. COX-2 immunostaining correlated most strongly with the chronic inflammation score (Fig. 4; $\mathrm{n}=$ $\left.20, \mathrm{r}^{2}=0.78, p<0.001\right)$. There was a correlation for those subjects who were $H$. pylori infected $\left(\mathrm{n}=10, \mathrm{r}^{2}=0.70, p=\right.$ $0.024)$, as well as for those who were uninfected $(\mathrm{n}=10$, $\left.\mathrm{r}^{2}=0.74, p=0.014\right)$.

\section{DISCUSSION}

Helicobacter pylori infection was associated with significant acute and chronic antral inflammation that was reduced by eradication of the organism, as expected. Our studies

Table 1. COX-2 Expression and Acute (A) and Chronic Inflammation (C) Scores

\begin{tabular}{ccccccccc}
\hline & \multicolumn{3}{c}{ H. pylori Infected } & & \multicolumn{3}{c}{ H. pylori Eradicated } \\
\cline { 2 - 3 } \cline { 6 - 8 } Patient & \% COX-2 & A & C & & \% COX-2 & A & C \\
\hline 1 & 38 & 3 & 3 & & 23 & 0 & 1.5 \\
2 & 40 & 2 & 4 & & 13 & 0 & 2 \\
3 & 41 & 3 & 3 & & 11 & 0 & 1 \\
4 & 5 & 1 & 1 & & 0 & 1 \\
5 & 39 & 1 & 3 & & 39 & 0 & 3 \\
6 & 46 & 3 & 3 & & 20 & 0 & 2 \\
7 & 11.5 & 1 & 2 & & 17 & 0 & 2 \\
8 & 12 & 2 & 3 & & 11 & 0 & 1 \\
9 & 50 & 3 & 3 & & 12 & 0 & 2 \\
10 & 51 & 1 & 3 & & 34 & 0 & 2 \\
\hline
\end{tabular}

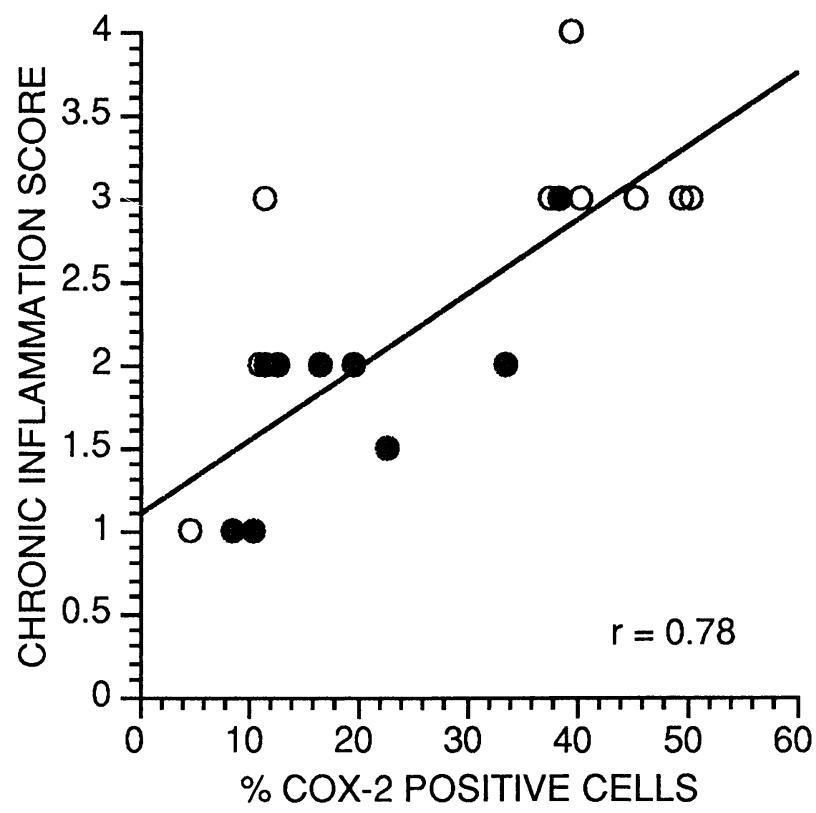

Figure 4. Correlation of COX-2 immunostaining with the chronic inflammation score for the entire sample $\left(\mathrm{n}=20, \mathrm{r}^{2}=0.78, p<\right.$ 0.001).

demonstrated immunohistochemical detection of COX-2 protein in antral epithelial cells, in addition to associated mononuclear cells, in the presence of $H$. pylori infection. In addition, parietal cells also exhibited COX-2 protein. Expression was reduced, but not eliminated, in the epithelium after successful eradication of $H$. pylori. Despite the reduction in COX-2 expression after $H$. pylori eradication, expression of COX-2 in epithelial cells remained and strongly correlated with the extent of the chronic inflammatory cell infiltrate.

Recently, other investigators have also reported the induction of gastric mucosal COX-2 expression with $H$. pylori infection. In a sample of $H$. pylori-infected and -uninfected patients, investigators in Hong Kong (8) confirmed only patchy COX-2 expression in superficial foveolar and glandular cells and mononuclear cells in the lamina propria. In H. pylori-infected mucosa, there was a significant increase in epithelial and mononuclear cell expression of the protein. However, by incubating antral mucosal biopsies from patients with $H$. pylori infection with highly selective COX-1 and COX-2 inhibitors, investigators from Nottingham, U.K. noted that the increase in prostaglandin production in $H$. pylori-infected mucosa was due primarily to the COX-1 isoform (9). These same investigators noted COX-2 expression predominantly in parietal cells in uninflamed mucosa and induction of COX-2 expression in macrophages, myofibroblasts, and endothelial cells in proximity to gastric ulcers (10). Parietal cell immunostaining for COX-2 was also noted in our study. The physiological implications of COX-2 in parietal cell function is not known, nor has the 
effect of highly selective inhibitors on acid secretion been characterized.

The increased COX-2 expression previously noted in healing ulcers may represent an essential reparative component in the inflammatory process. This is consistent with the observation that selective COX-2 inhibitors impaired ulcer healing (4). Alternatively, this increased COX-2 expression may not only reflect an important component of healing, but may represent a sequela of the inflammatory process with potentially ominous outcome. The association of induction of COX-2 expression with $H$. pylori infection, in addition to its presence in malignant gastric (6) and its well-characterized role in colon carcinogenesis (11), suggests that the induction of COX-2 may be an important mechanism in $H$. pylori-associated carcinogenesis. Because the development of $H$. pylori-related duodenal ulcer disease and gastric cancer occur through distinct pathways, the impact of COX-2 induction may have different implications in patients at risk for either outcome of the infection. Long-term studies on the impact of $H$. pylori eradication on the chronic inflammation and associated induction of COX-2 expression and subsequent cancer risk are needed to address this important question. Consistent with the observation that aspirin and NSAID use is associated with a reduction in gastric cancer risk (12), our work also suggests that selective COX-2 inhibitors should be studied with regard to chemoprevention of gastric cancer.

The relationship between $H$. pylori infection and nonsteroidal antiinflammatory drug (NSAID)-associated ulcer disease remains controversial. Whether $H$. pylori is a risk factor for the development of an NSAID ulcer remains controversial, although in some prospective studies of NSAID users, H. pylori infection was identified as a risk factor for the development of ulcers. Patients with evidence of current or past ulceration should have $H$. pylori eliminated; however, their risk for NSAID ulcer development may persist. Provocative recent data have suggested eradication of $H$. pylori infection can significantly reduce the short-term incidence of ulcers in NSAID users (13). In a prospective trial from Hong Kong, 92 patients were randomized to eradication therapy before naproxen for $8 \mathrm{wk}$. The number of endoscopic ulcers was significantly reduced by successful $H$. pylori eradication, and the numbers of symptomatic and bleeding ulcers were also decreased. These data, if confirmed by additional studies, support the potential utility of an aggressive $H$. pylori diagnostic and treatment approach in all NSAID users. However, a note of caution has been raised by investigators examining the role of $H$. pylori in the management of ulcers in NSAID users with omeprazole. In these studies (14), H. pylori infection was associated with improved efficacy of proton pump inhibitor therapy to reduce gastroduodenal injury. A number of explanations for this observation were proposed by the authors, including the suggestion that $H$. pylori-associated prostaglandin production may be beneficial in this clinical circumstance. This note of caution, in addition to the observation that a selec- tive COX-2 inhibitor could impair ulcer healing in rats, suggests that additional studies on the role of $H$. pylori infection on gastric prostaglandin physiology are needed.

In summary, our work has demonstrated that the $H$. pylori-infected stomach, in contrast with normal gastric epithelium, is associated with marked expression of COX-2 protein that is reduced after successful eradication therapy. Because chronic inflammation correlated best with protein expression, our results support additional studies on the timecourse of $H$. pylori-induced COX-2 message and protein expression, as inflammation may persist for months after $H$. pylori eradication. Since submission of this work, H. pylori has been demonstrated to directly induce COX-2 message and activity in a gastric mucosal cell line (15). Given the diverse implications of $H$. pylori infection, the clinical implications of $\mathrm{H}$. pylori-induced COX-2 expression deserve further study.

Reprint requests and correspondence: James M. Scheiman,
M.D., Associate Professor of Medicine, Division of Gastroenter-
ology, Taubman Center Box 0362, University of Michigan Med-
ical Center, Ann Arbor, MI 48109.
Received Aug. 3, 1998; accepted Jan. 11, 1999.

\section{REFERENCES}

1. Scheiman JM. NSAIDs, cytoprotection, and gastrointestinal injury. Gastroenterol Clin N Am 1996;25:279-98.

2. Crofford LJ. COX-1 and COX-2 tissue expression: Implications and predictions. J Rheumatol 1997:(suppl 49)24:15-9.

3. Kargman S, Charleson S, Cartwright M, et al. Characterization of prostaglandin $\mathrm{G} / \mathrm{H}$ synthase 1 and 2 in rat, dog, monkey and human gastrointestinal tracts. Gastroenterology 1996;111: 445-54.

4. Mizuno H, Sakamoto C, Matsuda K, et al. Induction of cyclooxygenase 2 in gastric mucosal lesions and its inhibition by the specific antagonist delays healing in mice. Gastroenterology 1997;112:387-97.

5. Infection with Helicobacter pylori. In: IARC Working Group on the Evaluation of Carcinogenic Risks to Humans, Schistosomes, Liver Flukes and Helicobacter pylori. IARC monographs on the evaluation of carcinogenic risks to humans. Vol. 61. Lyon, France: International Agency for Research on Cancer, 1994:177-240.

6. Ristimaki A, Honkanen N, Jankala H, et al. Expression of cyclooxygenase-2 in human gastric carcinoma. Cancer Res 1997;57:1276-80.

7. Elta GH, Scheiman JM, Barnett JL, et al. Long-term follow-up of Helicobacter pylori treatment in non-ulcer dyspepsia patients. Am J Gastroenterol 1995;90:1089-93.

8. To KF, Chan FKL, Leung WK, et al. Expression of cyclooxygenase $1(\mathrm{COX}-1)$ and 2$)(\mathrm{COX}-2)$ in $H$. pylori associated gastritis. Gastroenterology 1998;114:A310.

9. Jackson LM, Wu K, Mahida YR, et al. COX-1 expression in human gastric mucosa infected with Helicobacter pylori: Constitutive or induced? Gastroenterol 1998;114:A160.

10. Wu KC, Jackson L, Mahida YR, et al. Cyclooxygenase (COX1)-and 2 in human gastric mucosa: Constitutive expression by parietal cells and induction of COX-2 in lamina propria cells proximal to ulcers. Gastroenterol 1998;114:A334.

11. Sheng H, Shao J, Kirkland SC, et al. Inhibition of human colon 
cancer cell growth by selective inhibition of cyclooxygenase-2. J Clin Invest 1997;99:2254-9.

12. Thun MJ. Aspirin, NSAIDs, and digestive tract cancers. Cancer Metastasis Rev 1994;13:279-83.

13. Chan FKL, Sung JJK, Chung SCC, et al. Randomised trial of eradication of Helicobacter pylori before non-steroidal antiinflammatory drug therapy to prevent peptic ulcers. Lancet 1997;350:975-9.
14. Hawkey CJ, Karrasch JA, Szczepanski L, et al. Omeprazole compared with misoprostol for ulcers associated with nonsteroidal antiinflammatory drugs. N Engl J Med 1998;338:72734.

15. Romano M, Ricci V, Memoli A, et al. Helicobacter pylori up-regulates cyclooxygenase- 2 mRNA expression and prostaglandin E2 synthesis in MKN 28 gastric mucosal cells in vitro. J Biol Chem 1998;273:28560-3. 